\title{
Quantum Computation with a One-Dimensional Optical Lattice
}

\author{
Jiannis K. Pachos* and Peter L. Knight \\ Blackett Laboratory, Imperial College London, Prince Consort Road, London, SW7 2BW, UK
}

(November 10, 2018)

\begin{abstract}
We present an economical dynamical control scheme to perform quantum computation on a one dimensional optical lattice, where each atom encodes one qubit. The model is based on atom tunneling transitions between neighboring sites of the lattice. They can be activated by external laser beams resulting in a two-qubit phase gate or in an exchange interaction. A realization of the Toffoli gate is presented which requires only a single laser pulse and no individual atom addressing.
\end{abstract}

PACS numbers: 03.75.Lm, 03.67.Lx, 42.50.-p

The rapid pace of experimental development in trapping and manipulating cold atomic gases in optical lattices $[1,2]$ has inspired a series of models for quantum information processing [3] and quantum computation [4-10]. Arrays of localized qubits in optical lattices possess a great potential for the realization of an atom register. Atomic trapping chips $[11,12]$ offer ways in which atomic arrays can be manipulated at the single-atom level [13]. In the spirit of this investigation we present a simple model for quantum computation based on an optical lattice with one atom per lattice site and coherent manipulation between two different atomic ground states. Encoding the qubits in those ground states it is possible to perform one qubit gates by Raman transitions and two qubit gates by tunneling transitions. Adiabatic time evolutions are employed to generate a controlled dephasing evolution or an exchange interaction between any two neighboring atoms while preventing multiple occupancy of atoms in one site. Alternatively, it is possible to realize the same gates by timing a fast non-adiabatic evolution with transient population in states with two atoms per site. This results in a significant speedup of the gates. These evolutions comprise a universal set of gates which may be realized relatively easy experimentally. Their main advantage derives from their simplicity and their speed compared to other proposals $[7,8]$. The present scheme overcomes the problem of dephasing along the lattice sites [14] by employing atoms as individual qubits. Moreover, it offers the exciting possibility to construct multi-qubit gates such as the Toffoli gate with minimal resources.

The proposed model consists of a one dimensional chain of atoms with two ground states. The latter are coupled to each other with Raman transitions via an excited level as depicted in Fig. 1(a). The atoms are trapped in two parallel in-phase optical lattices with polarization $\sigma_{+}$and $\sigma_{-}$. These modes, denoted by $a$ and $b$, can be generated by two counter-propagating laser beams with parallel linear polarization vectors [15]. Each mode can trap one of the atomic ground states $\left|g_{a}\right\rangle$ or $\left|g_{b}\right\rangle$. The periodicity of the lattice is given by $\lambda / 2$ where $\lambda$ is the wavelength of the laser beam from which the standing waves are created. The loading of the optical lattice with approximately one atom per site can be achieved by a phase transition from the superfluid phase of a BEC to the Mott insulator phase [16]. This is implemented by increasing the intensity of the standing wave laser beam beyond the critical point that separates the two phases. Fock states are obtained in each lattice site for sufficiently large intensities. The effective Hamiltonian describing the interactions that take place is given by

$$
\begin{aligned}
& H=-\sum_{i}\left(J_{i}^{a} a_{i}^{\dagger} a_{i+1}+J_{i}^{b} b_{i}^{\dagger} b_{i+1}+J_{i}^{R} a_{i}^{\dagger} b_{i}+\text { H.c. }\right) \\
& +\frac{U_{a a}}{2} \sum_{i} a_{i}^{\dagger^{2}} a_{i}^{2}+U_{a b} \sum_{i} a_{i}^{\dagger} a_{i} b_{i}^{\dagger} b_{i}+\frac{U_{b b}}{2} \sum_{i} b_{i}^{\dagger} b_{i}^{2} .
\end{aligned}
$$

The nonlinear terms $U_{a a}$ and $U_{b b}$ are produced by the collisions of atoms of the same species with each other while $U_{a b}$ denotes interspecies collisions. $J^{a}$ and $J^{b}$ are the couplings of the tunneling transitions and $J^{R}$ is the effective coupling of the two ground states produced by a Raman transition. In particular, consider the setup for the formation of a one dimensional lattice where the trapping is generated by a cavity mode giving the potential

$$
V_{0}(\mathbf{x})=-V_{0} \sin ^{2} k x \exp \left(-\frac{2 r^{2}}{L^{2}}\right)
$$

where $r$ is the transverse distance from the lattice axis and $L$ is the width of the Gaussian profile of the cavity. Within the harmonic approximation at the minima of the lattice potential the collisional coupling is given by $U \approx 4 a_{s} V_{0}^{3 / 4} E_{R}^{1 / 4} / \sqrt{\lambda L}$ where $a_{s}$ is the scattering length of the atomic collisions and $E_{R}$ is the atomic recoil energy while the tunneling rate is given by

$$
J \approx \frac{E_{R}}{2} \exp \left(-\frac{\pi^{2}}{4} \sqrt{\frac{V_{0}}{E_{R}}}\right)\left[\sqrt{\frac{V_{0}}{E_{R}}}+{\sqrt{\frac{V_{0}}{E_{R}}}}^{3} .\right.
$$

*jiannis.pachos@imperial.ac.uk 
According to $[17,18]$ the critical value for obtaining a phase transition between the superfluid and the Mott phase in one dimension is given by $U / J \approx 11.6$.

To realize a quantum computational scheme we employ the above transitions in the following way. In the initial state the system contains one atom per lattice site, e.g. in mode $a$. Taking the two modes to be in phase we denote by $i$ the site corresponding to both modes. The evolution of the system is governed by Hamiltonian (1) where we shall demand individual activation of each coupling. As seen in Fig. 1(a) population can be transported from mode $a$ to mode $b$ and back within site $i$ by performing a Raman transition between the two ground states $\left|g_{a}\right\rangle$ and $\left|g_{b}\right\rangle$. By encoding logical $|0\rangle$ and $|1\rangle$ in these states we can easily implement a general one qubit rotation.

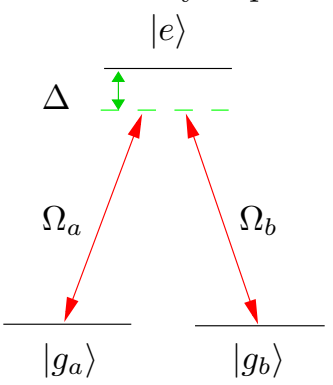

(a)

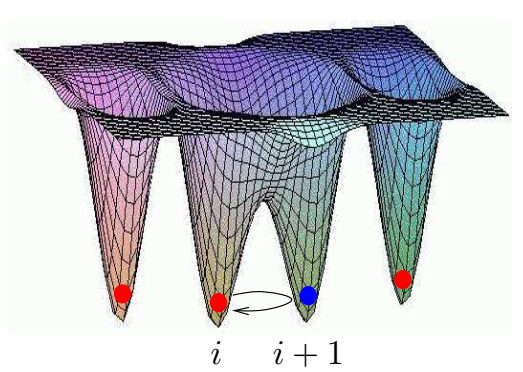

(b)
FIG. 1. (a) The atomic levels with two ground states coupled via an excited state by a Raman transition resulting to the $J^{R}$ coupling, and (b) the interaction between two neighboring sites where tunneling is activated by lowering the potential barrier between two neighboring sites.

To perform a two qubit gate we proceed as follows. We assume that the potential barriers between all sites are initially so high that no tunneling takes place throughout the optical lattice. Consider two neighboring sites $i$ and $i+1$ in modes $a$ and $b$ as depicted in Fig. 1(b). It is possible to lower the potential barrier between the two sites by employing an additional perpendicular standing laser field. Its minimum should be at the position midway between sites $i$ and $i+1$ and the waist of its Gaussian profile should not be larger than two lattice sites. This activates the tunneling between sites $i$ and $i+1$ in mode $a$ or $b$ depending on the circular polarization, $\sigma_{+}$or $\sigma_{-}$, of the applied laser field. In this way a hopping interaction is turned on between neighboring atoms that results eventually to a two-qubit phase gate or to an exchange interaction. To see how this is performed we need to consider in more detail the structure of Hamiltonian (1).

We shall take the induced tunneling couplings, $J$, to be much weaker than the collisional ones, $U$. In this regime the tunneling transitions from one site to the other are much weaker than the collision interactions between the atoms. We now consider the case with only one atom per lattice site, each prepared in a given superposition of the modes $a$ and $b$. By turning on and off the couplings $J$ such that at all times $J \ll U$, the evolution remains in the degenerate eigenspace of the collision terms. Indeed, the terms with coupling factors $U_{a a}$ and $U_{b b}$ are degenerate with respect to the occupation numbers $n=0$ and $n=1$. The state with two atoms occupying one site has an energy gap from the degenerate subspace given by $U_{a a}$ or $U_{b b}$. As a result the states with $n \geq 2$ can be adiabatically eliminated. In addition, having a large coupling $U_{a b}$ guarantees by the same reasoning that $n_{a}+n_{b}=1$ at all times. Simultaneous population of even one atom per mode in the same site has an energy gap proportional to $U_{a b}$ from the energetically lower states and hence is adiabatically avoided. As a result, the degenerate eigenspace spanned by $\left|n_{a}=1, n_{b}=0\right\rangle$ and $\left|n_{a}=0, n_{b}=1\right\rangle$ of every site is a well protected encoding space of the logical states $|0\rangle$ and $|1\rangle$. Hence, quantum information processing can be performed by quantum tunneling and by Raman transitions.

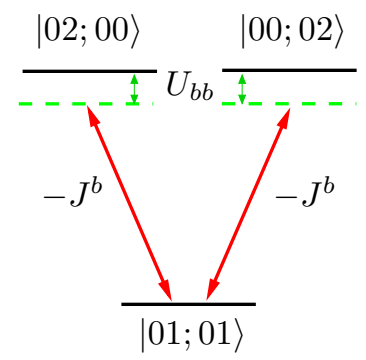

(a)

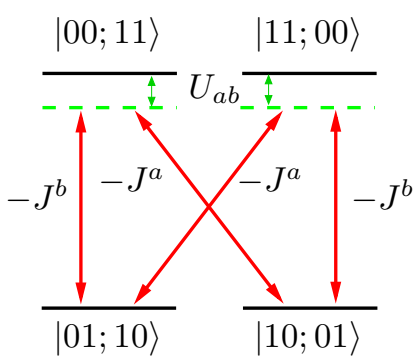

(b)
FIG. 2. Effective level scheme resulting from the tunneling and the collisional couplings. (a) The $V$-system that introduces a phase to the state $|01 ; 01\rangle$ due to its coupling with large detuning to the states $|02 ; 00\rangle$ and $|00 ; 02\rangle$. (b) The four level scheme with the two Raman couplings perform transitions between the ground states $|01 ; 10\rangle$ and $|10 ; 01\rangle$.

It is convenient to denote the states representing the atom population of the two sites as $\left|n_{a}^{1} n_{b}^{1} ; n_{a}^{2} n_{b}^{2}\right\rangle$. Let us initially consider the case where we lower the potential barrier between the two sites only in the $b$ mode. Then the logical two-qubit state $|11\rangle \equiv|01 ; 01\rangle$ couples to states with two atoms in one site, $|02 ; 00\rangle$ and $|00 ; 02\rangle$, by the Hamiltonian

$$
H_{1}=\left(\begin{array}{ccc}
0 & -J^{b} & -J^{b} \\
-J^{b} & U_{b b} & 0 \\
-J^{b} & 0 & U_{b b}
\end{array}\right) .
$$

This Hamiltonian corresponds to the level scheme presented in Fig. 2(a) that consists of a $V$-system. The effective "Rabi frequencies" of the lasers are both $-J^{b} / 2$ with an equal "detuning" $U_{b b}$. The states $|01\rangle$ and $|10\rangle$ obtain similar phase factors by an equivalent effect. If we compensate, in addition, for residual single qubit rotations of the form $\left(J^{b^{2}} / U_{a b}\right)|1\rangle\langle 1|$ on both qubits we can obtain a phase change only for state $|01 ; 01\rangle$ which is given by $\phi=2 \int_{0}^{T}\left(J^{b^{2}} / U_{a b}-J^{b^{2}} / U_{b b}\right) d t$. This is the case when $U_{b b}$ and $U_{a b}$ are much larger than $J^{b}$ and adiabaticity holds. Here $T$ denotes the overall time the coupling $J^{b}$ is turned on. In the logical space this evolution corresponds 
to the two-qubit phase gate $U=\operatorname{diag}(1,1,1, \exp i \phi)$ that together with general one qubit rotations results in universality.

It is easy to generalize this setup to realize three qubit gates like the control-control phase $\left(C^{2} P\right)$ and the Toffoli gate $\left(C^{2} N O T\right)$ with, in principle, the action of one laser pulse. Formally, the $C^{2} P$ gate gives a minus sign only to the logical state $|111\rangle$ and it can generate the Toffoli gate by $C^{2} N O T=(\mathbf{1} \otimes \mathbf{1} \otimes H) C^{2} P(\mathbf{1} \otimes \mathbf{1} \otimes H)$, where $H$ is a Hadamard gate. To generate $C^{2} P$ we lower the potential between three sites of mode $b$ in such a way that tunneling interaction is activated with coupling $J^{b}$ between neighboring sites and, for example, $\kappa J^{b}$ between next-to-neighboring sites. Without loss of generality and for simplicity we assume that $U_{a b} \gg U_{b b}$. For a laser pulse timed so that $\int_{0}^{T} J^{b^{2}} / U_{b b}=2 \pi n$ ( $n$ integer) and $12 \kappa \int_{0}^{T} J^{b^{3}} / U_{b b}^{2}=\pi$, second order perturbation theory shows that the state $|111\rangle$ acquires a minus sign, while the states $|110\rangle,|101\rangle$ and $|011\rangle$ perform a $2 \pi n$ rotation and the rest of the states are unaffected. This gives a $C^{2} P$ gate which can be transformed into a Toffoli gate by applying a Hadamard gate to the third qubit. Individual laser addressing is avoided by performing the Hadamard gate on all other qubits on the "right" of qubit three, simultaneously.

The entangling $\sqrt{S W A P}$ gate [19] can be implemented by engineering an exchange interaction between two neighboring sites. Let us consider the evolution of the degenerate states $|10 ; 01\rangle$ and $|01 ; 10\rangle$ when both of the tunneling rates $J^{a}$ and $J^{b}$ are activated. In the basis $|00 ; 11\rangle$, $|10 ; 01\rangle,|01 ; 10\rangle$ and $|11 ; 00\rangle$ the evolution is dominated by the Hamiltonian

$$
H_{2}=\left(\begin{array}{cccc}
U_{a b} & -J^{a} & -J^{b} & 0 \\
-J^{a} & 0 & 0 & -J^{b} \\
-J^{b} & 0 & 0 & -J^{a} \\
0 & -J^{b} & -J^{a} & U_{a b}
\end{array}\right) .
$$

At the same time the phase evolutions given by $H_{1}$ occur to both modes $a$ and $b$. Adiabatically eliminating the transitions outside the degenerate subspace provides an evolution similar to a Raman transition with the equivalent "Rabi frequencies" being $-J^{a}$ and $-J^{b}$ and with a "detuning" $U_{a b}$. The degenerate eigenspaces together with their couplings are depicted in the level scheme of Fig. 2(b). If the initial population is in the logical space and the tunneling rates are weak, then the adiabatic evolutions remains in this space resulting effectively in the Hamiltonian

$$
H_{\mathrm{eff}}=-I(|10\rangle\langle 01|+| 01\rangle\langle 10|)
$$

where $I=2 J^{a} J^{b} / U_{a b}$. The phase evolutions can be factorized out by choosing the couplings to satisfy the condition $J^{a^{2}} / U_{a a}+J^{b^{2}} / U_{b b}=\left(J^{a^{2}}+J^{b^{2}}\right) / U_{a b}$. In addition, one can compensate for residual single qubit rotations of both qubits of the form $\left(J^{a} / U_{a a}-J^{b^{2}} / U_{b b}\right)(|0\rangle\langle 0|-$ $|1\rangle\langle 1|)$ by applying properly tuned lasers.
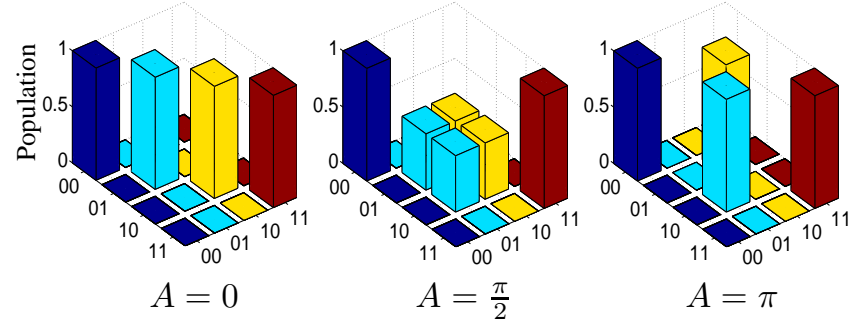

FIG. 3. Evolution of the density matrix elements $\left|\rho_{i j}\right|^{2}$ that comprise the two qubit space $\{|i j\rangle$; for $i, j=0,1\}$ for different values of the action $A=\int I d t$ that activates the exchange interaction.

In order to perform the $\sqrt{S W A P}$ gate we now turn on the coupling $I$, via the tunnel $J$ couplings for a certain time so that the action $A=\int I d t$ equals $\pi / 2$. For $A=\pi$ we obtain the $S W A P$ gate. The effective evolution of two qubits produced by $H_{\text {eff }}$ resulting in the exchange interaction is given in Fig. 3. The simulation was carried out with the full Hamiltonian (1) for a ratio of the couplings $J / U=10^{-2}$, which is well within the adiabatic weak coupling limit.

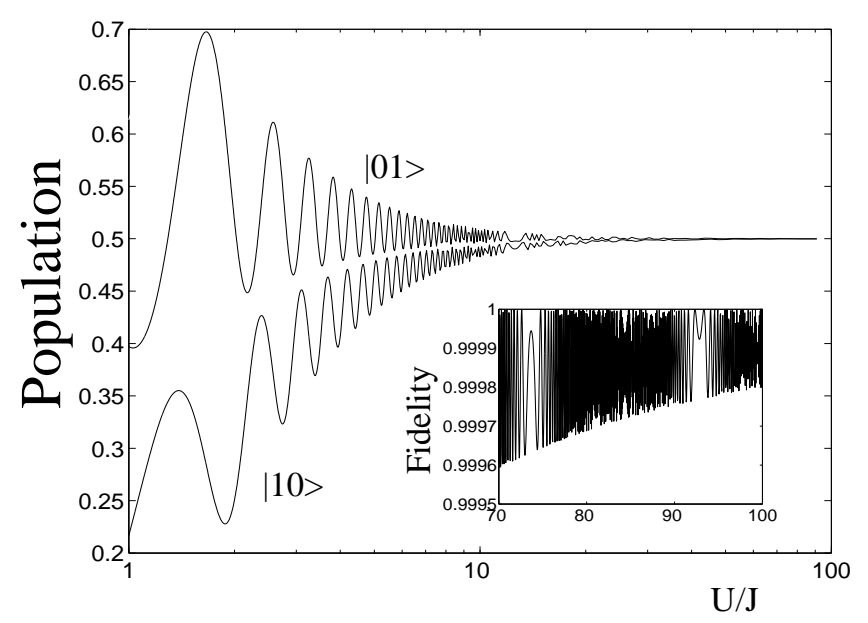

FIG. 4. The population of the states $|01\rangle$ (upper) and $|10\rangle$ (lower) when the tunneling interactions are activated with $A=\pi / 2$ on the initial state $|01\rangle$ as a function of $U / J$. The inset presents the fidelity of the exchange interaction for producing a maximally entangled state that deviates from unity by $4 \cdot 10^{-4}$ or less.

The condition of weak couplings $J$ with respect to the $U$ 's is necessary in order to adiabatically eliminate any evolution of the qubit states that would result in a population of two atoms in one lattice site. Indeed, by employing the effective exchange interaction to generate a maximally entangled state the fidelity of our procedure is $4 \cdot 10^{-4}$ or less away from unity. In Fig. 4 we see this fidelity for different ratios of $U$ and $J$. For $U \sim 10^{2} J$ it exhibits strong oscillations due to the large collisional couplings, but with a very small amplitude indicating the success of the adiabatic transition. This is paid for by 
obtaining slow gates. For currently measured collisional couplings [15] of the order of $1 \mathrm{kHz}$ a fidelity deviation from one less than $10^{-3}$ can be achieved if the duration of a macroscopic gate (e.g. a $\pi$-phase gate) is $T \sim 100 \mathrm{~ms}$. For these parameters the errors in the tunneling rate due to fluctuations of the laser intensity of the order of $10^{-3}$ contribute an acceptable gate error of the order of $10^{-3}$ or lower.

Alternatively, fast evolutions can be achieved by strong tunneling couplings. During these operations population is transferred into multi-occupancy states of the sites, but completely returns back to the logical space at certain times. Hence, by carefully controlling the timing of the tunneling procedure we obtain much faster gates. For example, the realization of a fast controlled phase-gate employs the same level scheme as in the previous. By timing the evolution so that $T_{n}=$ $2 \pi n / \sqrt{U_{b b}^{2}+8 J^{b^{2}}}$ we obtain the two qubit phase-gate with $\phi=\pi n\left[1+\left(U_{b b}-2 U_{a b}\right) / \sqrt{U_{b b}^{2}+8 J^{b^{2}}}\right]$. The condition $J^{b}=\sqrt{U_{a b}^{2}-m^{2} / n^{2} U_{b b}^{2}} /\left(2 \sqrt{2 m^{2} / n^{2}-1}\right),(m, n$ are positive integers with $m>n / \sqrt{2}$ ) guarantees that any possible transient population of two atoms per site is eliminated at the end of the gate.

Furthermore, it is possible to time the exchange interaction such that even for strong tunneling couplings there is zero population of states with two atoms per site regardless of their internal states (see Fig. 4). Consider for simplicity the couplings $J^{a}=J^{b}=$ $J$ and $U_{a a}=U_{b b}=U$. In this case the time interval for a completion of this evolution is given by $T_{n}=2 \pi n / \sqrt{U_{a b}^{2}+16 J^{2}}$ where we require $J=$ $\sqrt{U^{2}-m^{2} / n^{2} U_{a b}^{2}} /\left(2 \sqrt{4 m^{2} / n^{2}-2}\right)$. The condition for no-phase evolution of the states $|00\rangle$ and $|11\rangle$ is $U=2 U_{a b}$ if $m$ is an even integer. The resulting two-qubit gate $G$ is obtained at times $T_{n}$ and is given by $G\left(T_{n}\right)=$ $(|00\rangle\langle 00|+| 11\rangle\langle 11|)+\left[1+(-1)^{n} e^{-i / 2 U T_{n}}\right] / 2(|01\rangle\langle 01|+$ $|10\rangle\langle 10|)+\left[-1+(-1)^{n} e^{-i / 2 U T_{n}}\right] / 2(|01\rangle\langle 10|-H . c$.$) . This$ is in general a non-trivial gate that results together with any one-qubit gate in universality. These fast evolutions should be performed with couplings $J$ much smaller than the band gap of the trapped atoms in order to avoid population of higher vibrational modes of the optical trapping potential. In addition, the laser amplitude has to be stabilized in a more precise fashion than in the previously described adiabatic evolutions in order to achieve similar requirements in the gate precision. From such procedures we can achieve gate operation times of a few ms.

In conclusion, we have presented a simple scheme for performing two qubit gates based on adiabatic passages with tunneling interactions, which can effectively reproduce a phase gate or an exchange interaction. In addition, we have seen how three-qubit gates as the Toffoli gate can be easily generated without evoking individual atom laser addressing. The gate operation time can be greatly reduced by increasing the tunneling coupling and precise timing of the non-adiabatic evolution. In contrast to previous models for quantum computation on an optical lattice, the gates proposed here act locally between two neighboring qubits, while the rest of the lattice is non-interacting. In this way we are immune against the major experimental problem of dephasing of the lattice sites due to longitudinal irregularities of the lattice potential. In the future, it would be of much interest to study the possibility of performing the existing quantum algorithms in terms of common one-qubit gates and multi-qubit gates. As we have seen those are easy to realize within the present scheme, while individual one-qubit gates are experimentally much more difficult to implement.

Acknowledgments. We thank E. A. Hinds, M. Jones and A. Beige for discussions. This work was supported in part by the European Union and the U.K. Engineering and Physical Sciences Research Council.

[1] M. Greiner et al., Phys. Rev. Lett. 87, 160405 (2001); Greiner et al., Nature 415, 39 (2002).

[2] C. Orzel, et al., Science 291, 2386 (2001).

[3] S. Bose, "Quantum Communication Through an Unmodulated Spin Chain", quant-ph/0212041.

[4] D. Jaksch, et al., Phys. Rev. Lett. 82, 1975 (1999).

[5] R. Ionicioiu and P. Zanardi, Phys. Rev. A 66, 050301(R) (2002).

[6] E. Jané, et al., Quant. Inf. Comp. 3, (1) 15 (2003).

[7] L.-M. Duan, E. Demler, M. D. Lukin, "Controlling Spin Exchange Interactions of Ultracold Atoms in Optical Lattices", cond-mat/0210564.

[8] U. Dorner, D. Jaksch, M. Lewenstein, and P. Zoller, "Entangling strings of neutral atoms in $1 \mathrm{D}$ atomic pipeline structures", quant-ph/0212039.

[9] R. Raussendorf and H.-J. Briegel, Phys. Rev. Lett. 86, 5188 (2001).

[10] E. Charron, E. Tiesinga, F. Mies, and C. Williams, Phys. Rev. Lett 88, 077901 (2002).

[11] R. Folman et al., Adv. in Atomic, Molecular and Optical Physics, 48, 263 (2002).

[12] M. P. A. Jones, C. J. Vale, D. Sahagun, B. V. Hall, and E. A. Hinds, "Thermally induced spin flips above an atom chip", quant-ph/0301018.

[13] R. Long et al., Phil. Trans. Roy. Soc. (2003), in press.

[14] J. H. Denschlag, et al., J. Phys. B 35, 3095 (2002).

[15] O. Mandel, et al., "Coherent transport of neutral atoms in spin-dependent optical lattice potentials", condmat/0301169.

[16] D. Jaksch, et al., Phys. Rev. Lett. 81, 3108 (1998).

[17] M. P. A. Fisher et al., Phys. Rev. B 40, 546 (1989).

[18] W. Krauth, M. Caffarel, and J.-P. Bouchaud, Phys. Rev. B 45, 3137 (1992); K. Sheshardi et al., Europhys. Lett. 22, 257 (1993).

[19] D. Loss and D. P. DiVincenzo, Phys. Rev. A 57, 120 (1998). 